\title{
The biofilm inhibition and eradication activity of curcumin againts polymicrobial biofilm
}

\author{
Hasyrul Hamzah ${ }^{1}$, Triana Hertiani ${ }^{2,}$, Sylvia Utami Tunjung Pratiwi ${ }^{2}$, Titik Nuryastuti ${ }^{3}$, and Yosi Bayu Murti ${ }^{2}$. \\ ${ }^{1}$ Faculty of Pharmacy, Universitas Muhammadiyah Kalimantan Timur, Samarinda, Kalimantan Timur, 75124, Indonesia \\ ${ }^{2}$ Department of Pharmaceutical Biology, Faculty of Pharmacy, Universitas Gadjah Mada, Yogyakarta, 55281 Indonesia. \\ ${ }^{3}$ Department of Microbiology, Faculty of Medicine, Universitas Gadjah Mada, Yogyakarta, 55281 Indonesia.
}

\begin{abstract}
Curcumin is a polyphenol compound that is a member of the ginger family (Zingiberaceae), which has potential as an antibacterial, antifungal, and polymicrobial antibiofilm on the catheter. Still, its inhibitory activity and eradication of non-catheter polymicrobial antibiotics against $S$. aureus, $P$. aeruginosa, E. coli, and C. albicans have never been reported. The discovery of a candidate polymicrobial anti-biofilm drug is indispensable for overcoming infections associated with biofilms. This study aims to determine the inhibitory activity and eradication of curcumin on polymicrobial biofilms. Inhibition testing and eradication activity of polymicrobial biofilms were performed using the microtiter broth method. The effectiveness of curcumin on polymicrobial biofilms was analyzed using minimum biofilm inhibition concentration $\left(\mathrm{MBIC}_{50}\right)$ and minimum biofilm eradication concentration $\left(\mathrm{MBEC}_{50}\right)$. The mechanism of action of curcumin against polymicrobial biofilms is tested using scanning electron microscopy (SEM). Curcumin $1 \% \mathrm{~b} / \mathrm{v}$ gives biofilm inhibition activity in the mid-phase and maturation of $62.23 \% \pm 0.01$, $59.43 \% \pm 0.01$, and can eradicate polymicrobial biofilms by $55.79 \% \pm 0.01$ and not much different with nystatin drug control activity. The results also provide evidence that curcumin can damage the extracellular polymeric matrix (EPS) polymicrobial biofilms of $S$. aureus, P. aeruginosa, E. coli, and C. albicans and damage the morphology of polymicrobial biofilms. Therefore, curcumin can be developed as a candidate for new antibiofilm drugs against polymicrobial biofilms $S$. aureus, $P$. aeruginosa, E. coli dan C albicabs. Keywords: Biofilm, eradication, infection, polymicrobial bioflm, resistence
\end{abstract}

\section{Introduction}

Biofilms related to human infections are one of the problems in handling infections. Microbial diversity in polymicrobial biofilms results in chronic infections that are difficult to treat compared to monomicrobial biofilms. Biofilm-related infections are an increasing health problem worldwide, especially patients suffering from immune system disorders such as cancer, organ transplants, and malnutrition. There are not many antibiotics available that can effectively fight biofilm infections, which causes very high drug resistance.

Bacterial and fungal infections caused by biofilms are complicated to treat, to kill both bacteria and fungi in the form of biofilms requires 1000 times the dose of antimicrobials needed to achieve the same results as planktonic cells [1,2]. There is an increase in infections related to polymicrobial biofilms, including oral diseases, otitis media, sinusitis, diabetic wound infections, urinary tract infections (UTIs), and cystic fibrosis. Conditions related to polymicrobial biofilms are called complex and complicated because of the presence of several infectious agents. Infections due to polymicrobial biofilms provide additional challenges for treatment than monomicrobial biofilms [3]. Biofilms are acquiring clinically relevant importance and represent a problem that is still not sufficiently investigated, even less known concerning the behavior of communities of such mixed microorganisms [4].

Curcumin is a polyphenol compound that is a member of the ginger family (Zingiberaceae). Curcumin has been reported to have a broad spectrum, and It has biological activities such as anti-inflammatory, antioxidant, anticancer, antidiabetic, anti-allergic, antibacterial, and antiviral antiprotozoal, antifungal and polymicrobial antibiofilm on the catheter $[5,6]$. Curcumin has a high potential to be developed into antibiotics against $S$. aureus and other bacteria strains [7]. curcumin is beneficial in providing inhibitory activity against mono - species biofilms of $\mathrm{C}$. albicans by inhibiting protein synthesis so that it can result in inhibition of microbial growth [8].

Until now, no one has reported the inhibitory activity and eradication of curcumin against polymicrobial biofilms. With this research, we highlight the importance of studying complex biofilms that reflect microbial colonies found in nature compared to singlespecies biofilms representing only one case study in a laboratory. Specifically, we focus on the formation of

* Corresponding author: hertiani@ugm.ac.id 
polymicrobial biofilms $S$. aureus, P. aeruginosa, E. coli, and C. albicans.

\section{Material and methods}

\subsection{Material}

Materials used were Curcumin compound from the isolation of (Curcuma longa Linn.). Other elements include the following: crystal violet (Merck, Germany), ethyl acetate (Merck, Germany), Brain heart infusion (Oxoid) (Merck, Germany), RPMI 1640 (SigmaAldrich), catheter, ethanol $95 \%$ (Merck, Germany), nystatin, chloramphenicol (Sigma-Aldrich, Germany).

\subsection{Equipment}

Laminar Air Flow, incubator (IF-2B) (Sakura, Japan), micropipette pipetman (Gilson, France), multichannel micropipette (Socorex, Swiss), microplate flat-bottom polystyrene 24 well (Iwaki, Japan), microtiter plate reader (Optic Ivymen System 2100-C, Spain), spectrophotometry (Genesys 10 UV Scanning, 335903) (Thermo Scientific Spectronic, USA), autoclave (Sakura, Japan), incubator with orbital shaker $\mathrm{S} 1500$ (Stuart, UK), analytical scales (AB204 -5, Switzerland).

\subsection{Microbial preparation}

Staphylococcus aureus, Escherichia coli, Pseudomonas aeruginosa, are all grown within $24 \mathrm{~h}$ at $37^{\circ} \mathrm{C}$ in BHI media (Brain Heart Infusion). The fungus Candida albicans was produced for $72 \mathrm{~h}$ at $37^{\circ} \mathrm{C}$ temperatures in Sabouraud Dextrose Broth (SDB). 600 optical density from culture microbes is adjusted to 0.1 equivalent to McFarland standard $0.5-1.5 \times 10^{8} \mathrm{CFU} \mathrm{mL}^{-1}$ ), it is diluted in new growth media to $\mathrm{OD}_{600} 0.01$ for bacteria and $\mathrm{OD}_{520} 0.38$ for $C$. albicans.

\subsection{Polymicrobial biofilm formation inhibition assay in Vitro}

Minimum Biofilm Inhibition Concentration (MBIC) for the polymicrobial biofilm C. albicans ATCC 10231, $P$. aeruginosaATCC 27853, S. aureus ATCC 25923, E. coli ATCC 25922 was determined using the broth microdilution method. Polymicrobial suspensions were made from overnight cultures grown in BHI medium. The cells were harvested and washed twice with PBS, and the turbidity of the suspension was adjusted to 0.5 McFarland standard $\left(1 \times 10^{8} \mathrm{CFU} \mathrm{mL}^{-1}\right)$ in BHI [9]. The test solution's concentration was arranged in a polystyrene microtiter plate flat base 96 wells containing $200 \mu \mathrm{L}$ BHI medium. The final concentration of the test composition from $0.125 \%$ to $1 \% \mathrm{~b} / \mathrm{v}$ ). Nystatin and Chloramphenicol (at final concentrations ranging from $0.125 \%$ to $1 \% \mathrm{~b} / \mathrm{v}$ ) were used as positive control. The plates were then incubated at $37^{\circ} \mathrm{C}$ for $24 \mathrm{~h}$ and $48 \mathrm{~h}$. Supernatants from each well were poured, and planktonic cells were removed by washing with sterile PBS. The biofilm formation was stained using crystal violet and was read using a microtiter plate reader (Multisect Spectrum, Thermo Electron, Vantaa, Finland) at $595 \mathrm{~nm}$ [10].

\subsection{Polymicrobial biofilm eradication assay in Vitro}

The The effect of curcumin was also examined on previously formed polymicrobial biofilms of $C$. albicans polymicrobial biofilm ATCC 10231, $P$. aeruginosa ATCC 27853, S. aureus ATCC 25923, and E. coli ATCC 25922 Biofilms were inoculated in polystyrene microtiter plate like the one described above. After incubation at $37^{\circ} \mathrm{C}$ for $48 \mathrm{~h}$, the culture supernatant from each well was poured, and planktonic cells were removed by washing with PBS. Biofilm cells are exposed to various concentrations of test compounds (final concentration ranged from $0.125 \% \mathrm{~b} / \mathrm{v}-1 \% \mathrm{v} / \mathrm{b}$ ) and then incubated at $37{ }^{\circ} \mathrm{C}$ for $48 \mathrm{~h}$. Nystatin and chloramphenicol at the final concentration start from $0.125 \% \mathrm{~b} / \mathrm{v}-1 \% \mathrm{~b} / \mathrm{v}$ was used as positive control. After incubation, the plate was poured and washed three times, with $200 \mathrm{~mL}$ PBS to eliminate obedience cells. Biofilm reduction was quantified by the reduction of crystal violet test and was read using a microtiter plate reader (Multisect Spectrum, Thermo Electron, Vantaa, Finland) at $595 \mathrm{~nm}[11,12]$.

\subsection{Biofilm structure documentation using SEM}

Cells were grown directly on the slipcover or catheter and incubated at $37^{\circ} \mathrm{C}$ for $24 \mathrm{~h}$ for the middle phase. Test compounds are known to give $\mathrm{MBIC}_{50} / \mathrm{MBEC}_{50}$ activity at $0.25 \% \mathrm{~b} / \mathrm{v}$. After the mid-phase biofilm is formed, the coverslip is carefully washed with $1 \%$ PBS twice, followed by washing $2 \%$ paraformaldehyde, $2 \%$ glutaraldehyde, sodium cacodylate $0.15 \mathrm{M}$ and the sample is prepared to be observed under SEM. Sputter specimens are coated with layers gold, and samples were kept under JEOL Scanning electron microscopy (SEM)JSM-6400. The image is processed using Photoshop software [3].

\subsection{Statistical methods}

All experiments were performed in triplicate, and the results were expressed as the mean, standard deviation. Statistical analyses of the differences between the means of experimental groups were performed by one-way ANOVA followed by posthoc Bonferroni test. $P$ values $<0.05$ were considered as significant.

\section{Results and discussion}

\subsection{Effect of curcumin against polymicrobial biofilm mid-phase (24h) and mature phase (48h)}

Polymicrobial biofilms are a mixture of several species of bacteria and fungi. In this study, we examine the potential of curcumin antibiofilm for inhibition of 
polymicrobial biofilms: $S$. aureus, $P$. aeruginosa, $E$. coli, dan $C$. albicans. These results indicate that curcumin can inhibit $50 \%$ of polymicrobial biofilm formation in the middle and maturation (Figure 1). These results prove that curcumin compounds can inhibit the attachment of microbes to the surface so that biofilms' structure is disrupted.

In the mid-phase $1 \%$ curcumin compound was able to provide the activity of $78.50 \pm 0.01$ and was better than the activity provided by the control drug nystatin 1 $\%$ at $55.70 \% \mathrm{~b} / \mathrm{v} \pm 0.01$ and the control drug chloramphenicol $1 \quad \% \quad \begin{array}{cccccccc}6 & 0.82 & \% & \mathrm{~b} & / & \mathrm{v} & \pm & 0.01\end{array}$ (Figure 1). The $\mathrm{MBIC}_{50}$ value in the middle phase is at a level of $0.25 \% \mathrm{~b} / \mathrm{v}$.

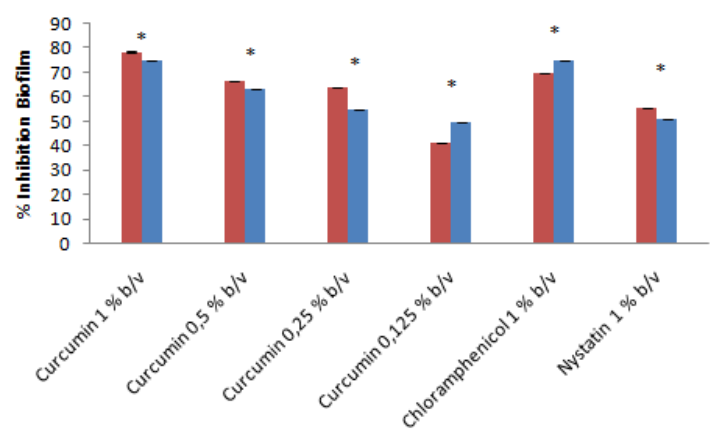

Fig. 1. Inhibition percentage of Curcumin on polymicrobial biofilm. (red bar: middle-phase, blue bar: maturation-phase, ${ }^{*}$ ) significance correlation $(P$ value $<0.05)$

In the maturation phase, there is a decrease in activity compared to the middle phase. This is because the growth time of biofilms at this phase is longer. The biofilm defense system is highly structured and forms cell communication between bacteria that form 3dimensional biofilms so that bacteria synergistically form more dense and complex defenses. Curcumin compounds in the maturation phase were only able to provide activity of $74.94 \% \pm 0.031$ and better than the activity given by the control drug nystatin $1 \%$ of $50.98 \% \mathrm{~b} / \mathrm{v} \pm 0.03$, and not too much different from the activity given. by control drug chloramphenicol $1 \%$ $74.72 \% \mathrm{~b} / \mathrm{v} \pm 0.01$ (Figure 1). $\mathrm{MBIC}_{50}$ value in the maturation phase is at the level of $0.25 \% \mathrm{~b} / \mathrm{v}$.

Biofilms produce thick and complex structures in the maturation phase compared to the middle phase, which can be seen from the mucus layer provided in the more mature and dense maturation phase attached to the wells. Therefore, biofilm in the maturation phase causes difficulty in penetrating the microbial antibiotics wrapped in biofilms because of the EPS matrix. These results are consistent with [13] biofilms in the ripening phase and are more difficult to penetrate than biofilms in the middle phase. The above account is also by the research of [2], which states that antimicrobial agents will have more difficulty penetrating biofilms' defenses in the maturation phase. Inter-species interactions cause colonization and infection dynamics and several other responses $[14,15]$. These results also provide the latest information about the natural compound curcumin has antibiofilm activity which has better polymicrobial antibiofilm activity than the control drugs nystatin and chloramphenicol

\subsection{Curcumin eradication activity against polymicrobial biofilms}

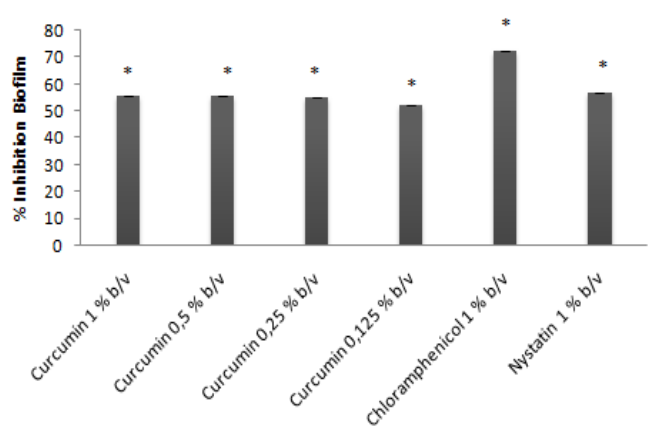

Fig. 2. Eradication activity of Curcumin against polymicrobial biofilms. *) significance correlation (P value $<0.05$ )

The research results provide evidence that the curcumin compound is capable of eradicating $S$. aureus, $P$. aeruginosa, E.coli, and C. albicans polymicrobial biofilms. The curcumin compound eradicated polymicrobial biofilm activity at $55.79 \pm 0.01$ while the control drug chloramphenicol was $72.63 \pm 0.01$ and nystatin $56.66 \pm 0.01$ (Figure 2).

These results indicate that curcumin compounds' activity in eradicating polymicrobial biofilms has decreased compared to the inhibitory activity given in the middle and maturation phases. This is because polymicrobial biofilms have a more extended growth period than in the intermediate and maturation phases, so the resulting EPS matrix is more complicated. These results are consistent with the research conducted by [14], which said that the EPS matrix in biofilms could help microbial cells last longer than if they are in planktonic conditions so that it can help the formation of microcortium of various microbial species to complete the degradation process. This stage of biofilm is in step 4 so that the continuity of biofilms is more organized and structured. Biofilms' ability to form such three dimensions makes their environment more difficult to penetrate by antimicrobial components because biofilms can form defenses that are strong enough and well organized to meet their individual needs.

This is by the statement of [16], which states that the formation of biofilms from microbial species both bacteria (Gram-positive and Gram-negative) as well as fungi mainly from the genus Candida albicans responsible for the emergence of disease in humans. Some literature explains that bacteria can synergistically form biofilms with other bacterial species and that the biofilm's physical and physiological structure becomes thicker and more durable $[17,18]$. 


\subsection{The result of Scanning Electron Microscopy (SEM) Polymicrobial Biofilm}
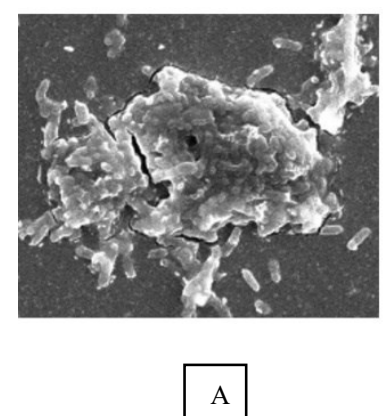

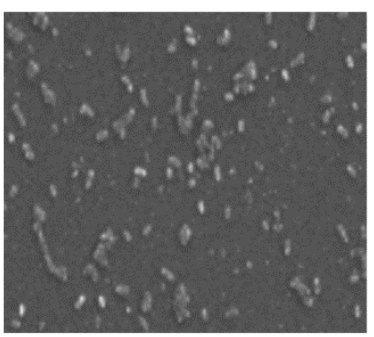

$\mathrm{B}$
Fig. 3. biofilm $S$. is taken using Scanning Electron Microscopy (SEM) (a) before giving Curcumin, (b) after administration of Curcumin compounds.

The SEM results show that the untreated polymicrobial biofilm shows the density of cells that are dense, structured, and formed EPS produced by polymicrobial biofilms (Figure 3a). In some literature, it is mentioned that bacteria synergistically form biofilms with other bacterial species, and the physical and physiological structure of biofilm is thick and robust $[12,17]$. The biofilm formation process also involves several bacteria, including the QS system and the two-component regulation system, both of which interact with each other, especially during EPS production [19, 20].

The results of SEM polymicrobial biofilms that have been given curcumin compound $0.25 \% \mathrm{~b} / \mathrm{v}$ indicate shrinkage, EPS matrix damage, and cells becoming lysis (Figure 3b). This is due to an increase in cell membrane permeability and to degrade mature biofilms by killing biofilms embedded in biofilms. These results are consistent with the statement made by [21] an increase in cell membrane permeability will facilitate the entry of components such as antibacterial into cells that will cause cell damage. According to [22], curcumin compounds can reduce yeast cells' sticking. Curcumin can damage the polymicrobial EPS biofilm matrix on the catheter and reduce the density of polymicrobial biofilm cells [23]. Curcumin can reduce biofilm biomass C. albicans Curcumin can reduce the biomass of C. albicans biofilms [24].

\section{Conclusion}

Curcumin compounds can inhibit the growth of polymicrobial biofilms and have been shown to have eradication activity against polymicrobial biofilms. Therefore, the curcumin compound was developed as a candidate for a new antibiofilm agent against polymicrobial biofilms.

Authors want to extend their gratitude to the microbiology Laboratory in the Faculty of Pharmacy Universitas Gadjah Mada, Yogyakarta, Indonesia. The authors declare no competing interests.

\section{References}

1. R.M. Donlan, J.W. Costerton, Clinical microbiology reviews, 15, 2, 167-193 (2002).

2. H. Hamzah, S.U.T. Pratiwi, T. Hertiani, Indonesian Journal of Pharmacy, 29, 4, 214 (2018).

3. B.M. Peters, M.A. Jabra-Rizk, A.O. Graeme, J.W. Costerton, M.E. Shirtliff, Clinical microbiology reviews, 25, 1, 193-213 (2012).

4. E. de Alteriis, L. Lombardi, A. Falanga, M. Napolano, S. Galdiero A. Siciliano et al., Microbial Pathogenesis. 125, 189-195 (2018).

5. S. Bansal, S. Chhibber, J. Med. Microbiol., 59, 4, 429-437 (2010).

6. H. Hamzah, T. Hertiani, S.U.T. Pratiwi, B. Murti, T. Nuryastuti, Research Journal of Pharmacy and Technology, 13, 1, 377-382 (2020).

7. S-Y. Teow, K. Liew, S.A. Ali, AS-B. Khoo, S-C. Peh, Antibacterial Action of Curcumin against Staphylococcus aureus: A Brief Review [Internet]. Journal of Tropical Medicine. (2016) [cited 2019 Mar 20]. Available from: https://www.hindawi.com/journals/jtm/2016/2853 $\underline{045 /}$

8. M.S.A. Khan, I. Ahmad, J. Antimicrob. Chemother., 67, 3, 618-621 (2012).

9. C.G. Pierce, P. Uppuluri, S. Tummala, J.L. LopezRibot, J. Vis. Exp. 44 (2010).

10. S.U.T. Pratiwi, E.L. Lagendijk, T. Hertiani, Weert SD, M CA, J.J.V.D. Hondel, International Journal of Pharmaceutical Research, 12, 183-191 (2015).

11. H. Hamzah, T. Hertiani, S.U.T. Pratiwi, T. Nuryastuti, Majalah Obat Tradisional, 24, 2, 110 118 (2019).

12. S.U.T. Pratiwi, H. Hamzah, Research Journal of Pharmacy and Technology, 11, 5425-5430 (2020).

13. A. Kannappan, M. Sivaranjani, R. Srinivasan, J. Rathna, S.K. Pandian, A.V. Ravi, Journal of Medical Microbiology, 66, 10, 1506-1515 (2017).

14. R.M. Donlan, Emerging Infectious Diseases, 8, 9, 881-890 (2002).

15. M.M. Harriott, M.C. Noverr, Antimicrobial Agents and Chemotherapy, 54, 9, 3746-3755 (2010).

16. H. Hamzah, T. Hertiani, S.U.T. Pratiwi, Nuryastuti T. International Journal of Research in Pharmaceutical Sciences, 11, 4, 5797-5802 (2020).

17. G.G. Anderson, J.J. Palermo, J.D. Schilling, R. Roth, J. Heuser, S.J. Hultgren, Science. 301, 5629, 105-107 (2003).

18. S.E. Cowan, E. Gilbert, D. Liepmann, J.D. Keasling, Applied and Environmental Microbiology, 66, 10: 4481-4485 (2000).

19. T.R. de Kievit, Environ. Microbiol. 11, 2, 279-288 (2009).

20. H. Hamzah, T. Hertiani, S.U.T Pratiwi, T. Nuryastuti, Research Journal of Pharmacy and Technology, 11 (2020).

21. X. Li, Microbiology, 149, 2, 353-362 (2003). 
22. H. Alalwan, R. Rajendran, D.F. Lappin, E. Combet, M. Shahzad, D. Robertson, et al., The Anti-Adhesive Effect of Curcumin on Candida albicans Biofilms on Denture Materials. Front Microbiol [Internet]. 2017 Apr 20 [cited 2019 Mar 20];8. Available from: https://www.ncbi.nlm.nih.gov/pmc/articles/PMC5 397414/

23. H. Hamzah, T. Hertiani, S.U.T Pratiwi, T. Nuryastuti, IJRPS, 11, 1, 830-835 (2020).

24. S.Z. Moghadamtousi, H.A. Kadir, P. Hassandarvish, H. Tajik, S. Abubakar, K. Zandi, BioMed Research International, 2014, 1-12 (2014). 